\title{
Self-Colocation: A Colocation Puzzle for Endurantists
}

\author{
Justin Mooney \\ University of Massachusetts Amherst \\ jmooney@umass.edu
}

[A version of this article is forthcoming in Synthese]

\section{Introduction}

Endurantism is the thesis that objects persist by being wholly located at distinct times. At any rate, this is one fairly standard way of characterizing the view. ${ }^{1}$ It's well-known that trouble arises when one attempts to spell out exactly what it means to be wholly located at some time, but I won't pursue those interesting issues here. I will simply take the wholly located at relation to be the primitive and fundamental location relation of Hudson (2005 and 2008a-b). ${ }^{2}$

Critics sometimes press endurantists on the problem of temporary intrinsics-i.e., the problem of how a single object like Socrates, who may be standing at one time and sitting at another, can have apparently incompatible intrinsic properties, like being straight and being bent. ${ }^{3}$ Many endurantists have tried to dissolve this problem by relativizing properties to times, or by adopting some cousin of this strategy. ${ }^{4}$

\footnotetext{
${ }^{1}$ Though see Gilmore (2004, ch. 2) for two different ways to draw the endurantist/perdurantist divide. The terms 'endurantism' and 'perdurantism' are due to Johnston (1984).

${ }^{2}$ For a critical discussion of Hudson's view, see Parsons (2008).

${ }^{3}$ Lewis $(1986,202-4)$ and Sider $(2001,92-8)$ are both influential presentations of the problem.

${ }^{4}$ See Haslanger (2003) and Kurtz (2006) for overviews of the options.
} 
But the relativizing-to-times trick soon gives way to a relativizingto-regions trick when time travel is introduced, because time travel permits a changing object to visit its past self, and so to have apparently incompatible properties at the same time. And more recently, even regionrelativization has suffered counterexamples in the form of 'self-colocation' or 'spooky coincidence' cases (Gilmore 2004, 190; Carroll 2011; Pruss 2017; Wasserman 2018, 199-203; Simon 20195). These are cases where "things...travel back in time and pass through their former selves" (Simon $2019,135)$. When the time-travelling object passes through itself, it can have apparently incompatible properties at the very same spatiotemporal region. So, endurantists have been pressed to seek alternatives to region relativization. ${ }^{6}$

While cases of self-colocation thus generate a particularly difficult variant of the problem of temporary intrinsics, in this paper I will argue that they also pose another problem for the endurantist. We will see that it is very difficult for the endurantist to say exactly what self-colocation is. In what follows, I develop this problem for endurantism and discuss a number of prima facie tempting solutions that, in my view, ultimately fail. Then I suggest a solution which I think is more promising, but which requires denying that apparent cases of self-colocation are genuine cases of selfcolocation. ${ }^{7}$

\section{A Colocation Puzzle}

Consider the following case. Casper the ghost hops into a time machine and travels back to a past time that we can dub t 1 to visit his younger self. In this way, Casper comes to be multilocated at t1. Though

\footnotetext{
${ }^{5}$ Simon cites APA comments by John Hawthorne as the source of these cases.

${ }^{6}$ These opening paragraphs follow Wasserman's (2018, ch. 6.3) outline of the temporary intrinsics dialectic.

${ }^{7}$ Self-colocation is one of many conceivable exotica at the intersection of mereology and location. For an overview, see Gilmore (2014) and the introduction to Kleinschmidt (2014).
} 
endurantists reject this view, it will sometimes be convenient to speak as though there are two distinct objects occupying the disjoint regions Casper occupies at t1: younger Casper, who has not yet travelled in time, and older Casper, who has just arrived at $\mathrm{t} 1$ from the future. It will also make for smoother going if we assume that Casper inhabits a Newtonian manifold, but nothing of substance hangs on this.

At $\mathrm{t} 1$, younger Casper is exactly located at a region $r 1$, and older Casper is exactly located at a region $r 2$, disjoint from $r 1$. But now imagine that, as time passes, older Casper leaves $\mathrm{r} 2$ and traces a continuous path through space until, at a later time, $\mathrm{t} 2$, he comes to occupy $\mathrm{r} 1$, the same region that is (still) occupied by younger Casper. Thus, older Casper apparently comes to be coincident with his past self. ${ }^{8}$

Similar cases have been described by other authors. Cody Gilmore $(2004,190)$ asks us to imagine an ethereal particle looping around a "selfintersecting timelike curve" to intercept itself; and Wasserman discusses a similar case involving a time-travelling boson (2018, 201; cf. 206-7). ${ }^{9}$ Simon (2019) has developed a particularly clever case featuring a commissurotomy patient.

Prima facie, these are all instances of a special variety of coincidence or colocation, namely: self-colocation. That is, they seem to be cases where an object colocates with itself. This is clearly Wasserman's view, and Simon's term "spooky coincidence" suggests that he, too, thinks of these cases as cases of self-colocation or self-coincidence. To see how natural this interpretation is, suppose (following other authors) that younger Casper is happy and older Casper is sad. Then it is tempting to think that a happy thing and a sad thing colocate at $\mathrm{r} 1$ at $\mathrm{t} 2$. But it also seems that both happy

\footnotetext{
${ }^{8}$ The case is adapted from Simon (2019), who in turn cites APA comments by John Hawthorne. I'm following Simon in speaking for convenience of younger Casper and older Casper as though they were distinct objects.

${ }^{9}$ For discussion of whether bosons can colocate, see the exchange between Cortes (1976), Barnette (1978), Ginsberg (1981), and Teller (1983).
} 
Casper and sad Casper are identical to Casper, so if happy and sad Casper colocate at $\mathrm{r} 1$ at $\mathrm{t} 2$, then Casper is apparently colocated with himself.

But what exactly is self-colocation? What does being self-colocated at some region amount to? This question leads to trouble for the endurantist. To see this, let's adopt "singular location" as a moniker for the ordinary, mundane way in which objects occupy spacetime. An object is singularly located only if it occupies some region, it is not colocated with anything else at that region, it is not multilocated at simultaneous regions, and so on. ${ }^{10}$ Since self-colocation is a variety of colocation, self-colocation is something different than mere singular location. But what is the difference? What, e.g., is the difference between Casper's being self-colocated at r1 at $\mathrm{t} 2$ and Casper's being singularly located at $\mathrm{r} 1$ at $\mathrm{t} 2$ ? This turns out to be a very difficult question for the endurantist to answer.

To see why, start by reflecting on colocation in general, rather than self-colocation in particular. Facts about colocation are not brute facts, but rather obtain in virtue of what I will call simple location facts, where a simple location fact is a fact that consists in a single object standing in a single instance of the location relation to a single spatiotemporal region (or a spatial region at a given time). For example, if two objects $a$ and $b$ are colocated at $r$, this seems to be in virtue of the simple location fact that $a$ is located at $r$ and the simple location fact that $b$ is located at $r$. And presumably, varying whether an object is colocated or only singularly located at some region will always require varying the simple location facts on which colocation depends. No difference in facts about colocation without a difference in facts about simple location. ${ }^{11}$

Since self-colocation is a kind of colocation, if Casper is self-colocated at $\mathrm{r} 1$ at $\mathrm{t} 2$, then this fact obtains in virtue of simple location facts, and these latter facts would be different in some way if Casper were merely singularly located at $\mathrm{r} 1$ at $\mathrm{t} 2 \mathrm{instead}$. What then are the facts in virtue of which Casper

\footnotetext{
${ }^{10}$ Among the further conditions one might want to add are these: it is not interpenetrating with any other objects and it is not an extended simple.

${ }^{11}$ Thanks to a referee for suggesting the use of a supervenience principle.
} 
is self-colocated at $\mathrm{r} 1$ at $\mathrm{t} 2$ ? Just as a pair of distinct objects $a$ and $b$ are colocated at $\mathrm{r}$ at $\mathrm{t}$ in virtue of the fact that $a$ is located at $\mathrm{r}$ at $\mathrm{t}$ and $b$ is located at $r$ at $t$, so it seems that Casper will be self-colocated at $r 1$ at $t 2$ in virtue of the fact that Casper is located at $\mathrm{r} 1$ at $\mathrm{t} 2$ and the fact that... Casper is located at $\mathrm{r} 1$ at $\mathrm{t} 2$. But those are the same fact, so all we really have is the fact that Casper is located at $\mathrm{r} 1$ at $\mathrm{t} 2$. And surely in that situation Casper is only singularly located at $\mathrm{r} 1$ at $\mathrm{t} 2 .^{12}$

So, an initial attempt to spell out what self-colocation amounts to seems to expose it as incoherent instead. ${ }^{13}$ As a variety of colocation, selfcolocation must be different than singular location. And yet, there does not seem to be any difference. But the argument for the latter claim tacitly presupposed endurantism, for it assumed that Casper is wholly, rather than partly, located at $\mathrm{r} 1$ at $\mathrm{t} 2$. If we drop this assumption and adopt the perdurantist's ontology instead, then it turns out that there is a straightforward account of self-colocation available. ${ }^{14}$

\footnotetext{
${ }^{12}$ A referee suggests that cases like Casper's seem like successful counterexamples to the principle that facts about colocation obtain in virtue of simple location facts. Fair enough, but I do not share that intuition myself. Perhaps one reason to hesitate before concluding that cases like Casper's are successful counterexamples to the principle in question is the availability of an alternative interpretation of those cases as special cases of singular location - an interpretation that I will ultimately recommend to the endurantist below. Even at this stage of the discussion there is something to be said for this alternative interpretation. Self-colocation already differs from standard colocation in at least one of the same ways that singular location does: it involves only one object (various readers have made this or related points). And if we deny that self-colocation obtains in virtue of simple location facts, this will only further decrease the resemblance between selfcolocation and standard colocation, while increasing the resemblance between self-colocation and singular location.

${ }^{13}$ For a related but distinct puzzle about motion, see Kleinschmidt (2017).

${ }^{14}$ Though I will speak only of perdurantism, what I say in this section could be reframed in terms of its close cousin, stage theory, which is defended by authors such as Hawley (2001) and Sider (2001).
} 
On perdurantism, younger Casper at $\mathrm{r} 1$ at $\mathrm{t} 2$ and older Casper at $\mathrm{r} 1$ at $\mathrm{t} 2 \mathrm{can}$ be understood as distinct proper parts of Casper. The first step to developing this proposal was taken by Sider (2001, 101ff.), who discusses an imaginary case in which he travels back in time to visit his younger self, so that there are two spatially separated Sider-like objects in the same room at once. Sider argues that the perdurantist should interpret these objects as distinct spatial parts of a single temporal part - spatial parts that are intrinsically just like ordinary temporal parts of Sider, and fail to qualify as temporal parts only because they exist simultaneously.

Applying this idea to the story of Casper, when older Caspar arrives from the future at $\mathrm{t} 1$, there are two spatially separated instantaneous ghostlike objects at $\mathrm{t} 1$, each of which is a proper part of Casper. At each moment after $\mathrm{t} 1$, there is another instantaneous pair of such objects, which are increasingly close together as older Casper approaches younger Casper, until complete coincidence occurs at $\mathrm{r} 1$ at $\mathrm{t} 2$. But should the perdurantist say that there really are two instantaneous ghostlike objects colocated at $\mathrm{r} 1$ at $\mathrm{t} 2$, or should she say that there is actually just one? If we continue to imagine that younger Casper is happy and older Casper is sad, then she should say that there are two. For one of the traditional advantages of perdurantism is that it allows for apparently incompatible temporary intrinsic properties like happy/sad to really be incompatible temporary intrinsic properties, never had by the very same object. So, in keeping with this perdurantist tradition, the perdurantist should say that there is a happy ghostlike object at r1 at t2 colocated with a distinct, sad ghostlike object at $\mathrm{r} 1$ at $\mathrm{t} 2 .{ }^{15}$

\footnotetext{
${ }^{15}$ What should the perdurantist say in a case where younger and older Casper are intrinsic duplicates? I think that she should say there is only one instantaneous ghostlike object at $\mathrm{r} 1$ at $\mathrm{t} 2$, because positing two would be ontologically extravagant. In the case where younger casper is happy and older Casper is sad, the persistence of younger Casper at $\mathrm{r} 1$ causes a happy ghostlike object to occupy r1 at t2, while the movement of older Casper toward r1 causes a sad ghostlike object to
} 
One might worry that this proposal will violate extensionality, which claims that any objects $x$ and $y$ share all of the same parts iff they are identical. For it might seem that the happy ghostlike object and the sad ghostlike object at $\mathrm{r} 1$ at $\mathrm{t} 2$ share all of the same parts, and so they cannot be construed as distinct proper parts of Casper without running afoul of extensionality - a consequence that most perdurantists won't accept. ${ }^{16}$

But the perdurantist can deny that the ghostly objects at $r 1$ at $t 2$ have all of the same parts. Since we are imagining ethereal objects that are disposed to interpenetrate, I think that the most natural way to interpret what is going on at moments where they occupy overlapping regions is that they spatially overlap without mereologically overlapping. So, when $t 2$ arrives, there are two instantaneous ghostlike objects colocated at r1 without sharing any parts. This will be a case of colocation by interpenetration rather than a case of the matter-sharing variety of colocation which perdurantists typically eschew anyway. ${ }^{17}$

On this account, the two instantaneous ghostlike objects at $\mathrm{r} 1$ at $\mathrm{t} 2$ don't share any parts in common, so there is no violation of extensionality. If these ghostlike objects are simples, then their respective improper parts are distinct, where this distinctness is plausibly a brute fact. (I take it the distinctness of any two or more simples is a brute fact.) So there is no

occupy $\mathrm{r} 1$ at t2. But if old and younger Casper are (say) both happy, then the more parsimonious proposal is that there is one happy ghostlike object at $\mathrm{r} 1$ at $\mathrm{t} 2$ whose existence at that location is overdetermined by the persistence of younger Casper and the motion of older Casper. There won't be any genuine colocation going on at $\mathrm{r} 1$ at $\mathrm{t} 2$, but it isn't clear that this is a cost, for it is in keeping with how the perdurantist handles (some) other coincidence cases. The typical perdurantist says that some regions which seem to be occupied by distinct, colocated objects are in fact occupied by a single, shared temporal part of two or more (non-colocated) objects.

${ }^{16}$ Thanks to a referee for this objection.

${ }^{17}$ Granted, allowing interpenetration entails either that supersunstantivalism is false, or that regions themselves can interpenetrate. Some perdurantists will not be happy with this, but others won't be bothered at all. I take it that this issue is largely independent of perdurantism itself. For more on interpenetration, see Gilmore (2014). 
violation of extensionality. ${ }^{18}$ If the ghostlike objects are composites of instantaneous parts of some kind, then those parts themselves are distinct (though interpenetrating), and so again there is no violation of extensionality. Either way, the perdurantist can say that there are two instantaneous ghostlike objects colocated at $\mathrm{r} 1$ at $\mathrm{t} 2$.

Now, if perdurantism is true, we often speak as if an object is located where any temporal part of that object is located. For example, we would say that I am sitting in this chair now even though only a small temporal part of me is sitting in (a small temporal part of) this chair now. Similarly, in cases like Casper's, where two parts of Casper that are intrinsically just like temporal parts colocate, it seems appropriate to say that Casper is colocated with himself. Therefore, the perdurantist can make sense of selfcolocation cases by interpreting them as cases where two or more parts of a persisting object that are intrinsically just like temporal parts colocate at some region. ${ }^{19}$

By contrast, the standard endurantist cannot say this, because her ontology does not include such things as temporal parts or objects intrinsically like temporal parts spread throughout a persisting object's career. So the challenge for the endurantist is to give us a coherent account of self-colocation without appealing to the perdurantist's resources. In the

\footnotetext{
${ }^{18}$ Anticipating either this response or at least a similar response, the referee worries that there will now be a violation of the Strong Supplementation axiom, which requires that, if one object $\mathrm{x}$ is not a part of another object $y$, then $\mathrm{x}$ has a part that doesn't overlap $\mathrm{y}$. But, while neither ghostlike object at $\mathrm{r} 1$ at $\mathrm{t} 2$ is part of the other, they do not violate Strong Supplementation because each ghostlike object does have a part that doesn't overlap the other, namely, it's improper part. Granted, these two improper parts are spatially coincident, but again, this doesn't entail that they mereological overlap; they may be interpenetrating.

${ }^{19}$ As a referee observes, there may also be other options open to the perdurantist for making sense of self-colocation, since she might be able to extend her treatment of permanent material coincidence cases to apparent self-colocation cases. The endurantist, on the other hand, will probably find this sort of approach no more appealing as an account of self-colocation than as an account of permanent material coincidence.
} 
next section, I will critically survey a number of ways the endurantist might try to accomplish this feat.

\section{$4 \quad$ Unsuccessful Endurantist Solutions}

First, it's worth exploring whether the endurantist can mimic the perdurantist's account of self-colocation but replace the perdurantist's temporal-part-like objects with something else that fills the same role. To pull this off, the endurantist would need to posit multiple Casper-shaped objects that are coincident with each other at $\mathrm{r} 1$ at $\mathrm{t} 2$, and which, though not identical to Casper, can be loosely spoken about as if they were. If the endurantist can find such objects, she could cast self-colocation as the coincidence of these objects at some region. ${ }^{20}$ Provided that there is no such coincidence in cases of singular location, self-colocation will differ from singular location.

What endurantist-friendly objects are there that could fill this role? Here's an idea. Some metaphysicians think that certain objects are constituted by other objects, and, moreover, that the former are constituted by different objects at different times. For example, it is common to hold that a statue can be constituted by different lumps of clay at different times; or that an organism can be constituted by different fusions of particles at different times; and so on. A time travelling object that visits its past self might therefore be constituted by different objects at the same time. Presumably, if I were to travel back in time to visit myself at a younger age, younger me would be constituted by a different fusion of particles than older me. Then these distinct fusions of particles could colocate. So, perhaps cases of self-colocation are cases where distinct objects at some time $t$, each of which constitutes one and the same further object at $t$, are colocated.

I don't think this is going to work. Not every object which could plausibly be self-colocated is also plausibly constituted by another object.

\footnotetext{
${ }^{20}$ Simon (2019) may have something like this in mind when he refers to Casper's 'two coincident manifestations' (135).
} 
Casper himself might be one example. What, if anything, constitutes a ghost? There doesn't seem to be any lump of material out of which a ghost is made. Nor is it typical to think of ghosts gaining and losing parts, which might otherwise tempt us to suggest that ghosts are constituted by, but not identical to, fusions of their parts. And even if ghosts are constituted by fusions of their parts, why think that they are constituted by different fusions at different times? If this business about ghosts seems silly, there are more serious cases. For example, surely mereologically simple, pointsized particles are not constituted by any further object, and yet one can construct spooky coincidence cases featuring particles (e.g. Gilmore 2004, 190; Wasserman 2018, 199-203).

Or consider a case like this. Imagine that I am constituted by a certain fusion of particles, $F$, at a region $r$ and time $t$. As time passes, I am constituted by different fusions of particles in succession, while the particles which are the parts of $\mathrm{F}$ each go their own way, becoming scattered throughout the biosphere. But then suppose that, by an enormous coincidence, I come to be constituted by $\mathrm{F}$ again at some later time, at the very instant I step into a time machine located at $r$ that immediately transports me back to $t$, where I colocate with myself. Now younger me and older me are colocated, but also constituted by the same fusion of particles, namely, F. Once again, there are not two distinct, colocated objects which constitute me at $t$, so the constitution strategy fails to account for this case of self-colocation. Therefore, the constitution strategy at best falls short of a general account of self-colocation.

Let's try again. Jeffrey Brower's (2010) neo-Aristotelian solution to the problem of temporary intrinsics may provide an endurantist substitute for person stages and their ilk in self-colocation cases. To explain how a persisting object can have apparently incompatible properties at different times, Brower hypothesizes that objects persist by successively colocating with momentary objects. For example, on Brower's view, there are objects such as seated Socrates and standing Socrates, which are distinct from each other and which are confined to the times at which Socrates is sitting and standing, respectively. Socrates himself persists by successively colocating 
with these objects. Thus, Socrates changes from sitting to standing by first colocating with sitting Socrates at $\mathrm{t} 1$, and then colocating with standing Socrates at $\mathrm{t} 2$.

Perhaps a friend of Brower's view could say that self-colocation occurs when a persisting object simultaneously colocates with two or more momentary objects which are themselves colocated. So, returning to Casper, and supposing again that younger Casper is happy and older Casper is sad, we might say that the momentary objects happy Casper and sad Casper are colocated at $\mathrm{r} 1$ at $\mathrm{t} 2$, and that Casper himself colocates with both of them at $\mathrm{t} 2$. In this way, Casper self-colocates.

But once again, I fear this won't account for all of the possible cases of self-colocation. For suppose that younger and older Casper do not have different moods (happy/sad), but rather are intrinsic duplicates. Then presumably there will be just one momentary object at $\mathrm{r} 1$ at $\mathrm{t} 2$, rather than two or more different, colocated momentary objects. If Casper is happy throughout his entire life, then there will be a happy Casper at $\mathrm{r} 1$ at $\mathrm{t} 2$, but no sad Casper colocated with happy Casper. But if Casper time travelled back to 2 and interpenetrated his past self at $r 1$, then presumably he is selfcolocated there in spite of there being no difference in properties like happy / sad. So, this account of self-colocation, like the constitution account above, doesn't accommodate all of the possible cases. ${ }^{21}$

Our attempt to mimic the perdurantist account of self-colocation is not going well. A different strategy we might try concedes that there is just one Casper-shaped object at r1 at t2, namely, Casper himself, but argues that Casper is somehow located at $\mathrm{r} 1$ at $\mathrm{t} 2 \mathrm{twice}$ over. ${ }^{22}$

\footnotetext{
${ }^{21}$ One might push back by resisting the claim that there would only be one momentary object at $\mathrm{r} 1$ at $\mathrm{t} 2$ in the case just described. Why couldn't there be two such objects which were intrinsically just alike- a happy Casper colocated with another happy Casper? I think this proposal suffers from a problem that I will press against duplication proposals below, by appealing to overdetermination.

${ }^{22}$ Thanks to a referee for this suggestion. Simon $(2019,124)$ uses the language of being located at a region twice over in his discussion of self-colocation cases, but it's unclear whether he means to be suggesting the strategy I pursue here under that description.
} 
One way to pursue this thought is to propose that Casper's being located at $\mathrm{r} 1$ at $\mathrm{t} 2$ occurs twice over in some hypertemporal or hyperspatial dimension. ${ }^{23}$ Then the difference between singular location and selfcolocation is a matter of how many hyper-locations there are at which Casper is located at $\mathrm{r} 1$ at $\mathrm{t} 2$ : if Casper is located at $\mathrm{r} 1$ at $\mathrm{t} 2$ at just one hyperlocation, or at no hyper-locations at all, then Casper is at most merely singularly located at $\mathrm{r} 1$ at $\mathrm{t} 2$; but if Casper is located at $\mathrm{r} 1$ at $\mathrm{t} 2$ at two or more hyper-locations, then Casper is self-colocated at $\mathrm{r} 1$ at $\mathrm{t} 2$.

Simon (2019) objects to proposals of this sort on the grounds that they are ontologically extravagant. Having argued that spooky coincidence may be nomically possible, he notes the implication that hyper-location proposals may require not merely that there are possible worlds containing hyperspace or hypertime, but that the actual world does, too.

Nor will it do any good to say that Casper is located at $\mathrm{r} 1$ at $\mathrm{t} 2$ at two moments of Casper's personal time. ${ }^{24}$ This strategy has been roundly criticized in related contexts (Sider 2001, 106; Carroll 2011; Wasserman 2018, 202; Simon 2019, 136, n. 23). The main problem is that personal time is defined using events in external time, and so it cannot be used to distinguish what is not already distinct. Unless Casper is already located at r1 at $\mathrm{t} 2$ twice over, there cannot be distinct moments of personal time at which Casper is located at $\mathrm{r} 1$ at $\mathrm{t} 2$.

But maybe appeals to such things as hyper-dimensions and personal times is a red herring. Why not postulate duplicate location facts without indexing them to distinct hyperlocations? This strategy comes in many forms. ${ }^{25}$ For example, we could postulate tropes or particular instances of the location relation, and then claim that in singular location, there is just one trope or instance of the located at relation uniting Casper to $\mathrm{r} 1$ at $\mathrm{t} 2$, but in a case of self-colocation there are two tropes or instances of the located at

\footnotetext{
${ }^{23}$ Hud Hudson is one prominent hyperspace and hypertime theorist. See especially Hudson (2005) and (2014).

${ }^{24}$ The notion of personal time was introduced by Lewis (1976).

${ }^{25}$ Thanks to a referee for pointing out the many forms that it could take.
} 
relation uniting Casper to $\mathrm{r} 1$ at $\mathrm{t} 2$. Those of us who reject tropes and their ilk might be happy instead with facts, states of affairs, or events. We could say that, in a case of singular location, there is just one fact, or state of affairs, or event, that consists of Casper's being located at $\mathrm{r} 1$ at $\mathrm{t} 2$, but that in a case of self-colocation there are two duplicate facts, or states of affairs, or events, that each consist of Casper's being located at $\mathrm{r} 1$ at $\mathrm{t} 2$.

This proposal suffers from a strange ontological extravagance that can be brought out best by considering classic cases of overdetermination. Imagine a subject, S, who is shot simultaneously by two snipers, each of whom has fired a fatal shot. In this case, two causal sequences eventuate in the death of S. And don't let the term 'overdetermination' distract you. It seems that even if the two causal sequences are indeterministic-if, for example, a quantum fluke might have caused either of the snipers to missnevertheless both causal sequences cause S's death. But it would be extravagant to suppose that, because two causal sequences eventuate in $S^{\prime} \mathrm{s}$ death, $\mathrm{S}$ suffers a kind of double-death, i.e., that there are two, duplicate, colocated events (or states of affairs, etc.) consisting of $S^{\prime}$ s dying.

I say the same about cases of self-colocation. In the case of Casper, there are two causal sequences that eventuate in Casper's being located at r1 at t2. The first is younger Casper's persisting at r1; the second is older Casper's moving from r2 toward r1. Whether deterministic or indeterministic, both of these causal sequences cause Casper to be located at $\mathrm{r} 1$ at $\mathrm{t} 2$. But just as it was extravagant to suppose that $\mathrm{S}$ suffered two colocated deaths when shot by two snipers, so it is extravagant to suppose that there are two colocated cases of Casper being located at $r 1$ at $t 2$ in our self-colocation case. So, this strategy is not a success either.

Let's change tactics once more. Perhaps the difference between selfcolocation and singular location lies in facts about Casper's history. We could say that Casper is self-colocated at $\mathrm{r} 1$ at $\mathrm{t} 2$ rather than singularly located at $\mathrm{r} 1$ at $\mathrm{t} 2$ in virtue of the historical fact that Casper arrived there via 
two different routes, the second time by travelling back in time and passing through his younger self. ${ }^{26}$

But I think that it is a mistake to look at times other than $\mathrm{t} 2$ to find what it is in virtue of which Casper is self-colocated at r1 at t2. To see why, we need to reflect once again on colocation in general, rather than selfcolocation in particular. Suppose a pair of objects, $x$ and $y$, are colocated at $r$ at $t$. As we saw above, it seems that all this requires is the simple location fact that $\mathrm{x}$ is located at $\mathrm{r}$ at $\mathrm{t}$, and the simple location fact that $\mathrm{y}$ is located at $r$ at $t$. And these facts do not seem to depend on what is going on at other times and places, or indeed, on anything extrinsic to $r$ at $t$. To see this, recall that simple location facts consist of a single object standing in a single instance of the location relation to a single spatiotemporal region or region at a time. Regions, the location relation, and at least some material objects are plausibly fundamental, so, by any recombination principle that allows fundamental entities to be freely recombined, the colocation of $\mathrm{x}$ and $\mathrm{y}$ at $\mathrm{r}$ at $\mathrm{t}$ is not going to require anything about where $\mathrm{x}$ and $\mathrm{y}$ were located at earlier times, or that either of them has time travelled to $r$ at $t$, or whether there even is anything outside of $r$ at $t^{27}$

So, facts about colocation at some time do not seem to obtain in virtue of facts about what is going on at other times. Since cases of selfcolocation are ipso facto cases of colocation, a suitable analysis of selfcolocation ought to allow that self-colocation at some time obtains only in virtue of facts about what is going on at that same time. Therefore, the strategy of appealing to Casper's history is not a plausible route to securing an account of self-colocation.

One could resist this argument by claiming that independence of facts about other times is a feature of paradigmatic cases of colocation, but not a feature of all cases, and in particular not cases of self-colocation. But this move is costly, because self-colocation cases already deviate from

\footnotetext{
${ }^{26}$ My thanks to the many readers who pressed versions of this objection.

${ }^{27}$ This argument is based on the recombinatorial arguments in Sider (2000) and Brzozowski (2008).

Cf. the recombinatorial arguments in McDaniel (2007a-b) and Saucedo (2011).
} 
paradigmatic cases of colocation in virtue of involving just one object, ${ }^{28}$ and the less these cases resemble paradigmatic cases of colocation, the less plausible it is to claim that these are instances of colocation at all.

\section{$5 \quad$ A More Promising Endurantist Solution}

Though we haven't yet found a solution for the endurantist, I think that the foregoing discussion suggests a more promising - and very different - way forward. In light of the failure of all the strategies we have considered, it's plausible that the history strategy gets this much right: the only difference between a mundane case in which Casper is singularly located at $\mathrm{r} 1$ at $\mathrm{t} 2$ without time-traveling (etc.), on the one hand, and the time travel case that has been the focus of our attention, on the other hand, is Casper's history. But as we just saw, this history is probably not enough to take what would otherwise be a mundane case of singular location and turn it into a case of self-colocation. So maybe what the endurantist should say is that, even in the time travel case, Casper is merely singularly located at $\mathrm{r} 1$ at $\mathrm{t} 2$.

More generally, I propose that the endurantist should interpret cases of time traveling objects that appear to colocate with themselves as cases of singular location in the midst of strange time travel adventures. The story goes something like this. At t1, Casper is multilocated. And when younger Casper begins to interpenetrate older Casper, they remain multilocated while they occupy distinct but overlapping regions. But at the moment when younger Casper comes to occupy r1 at $\mathrm{t} 2$, Casper is no longer either multilocated or interpenetrating himself. Rather, Casper is merely singularly located at $\mathrm{r} 1$ at $\mathrm{t} 2$. Now authors who have discussed these cases agree that, e.g., Casper will retain the properties of both older Casper and younger Casper even when he passes through himself. This means that Casper will have two different velocities when he is located at r1 at t2.

\footnotetext{
${ }^{28}$ May thanks to the various readers who made this point or closely related points.
} 
Therefore, at the next moment, $\mathrm{t} 3$, Casper will multilocate again, heading off in two different directions at once. ${ }^{29}$

The question remains how Casper can have apparently incompatible properties at the same place and time. For suppose younger Casper has velocity v1 and older Casper has a distinct velocity v2. It's tempting to reason that, if younger Casper has velocity v1, then he doesn't have velocity v2 like older Casper does, and so by Leibniz's law younger Casper is distinct from older Casper. Of course, this is not a unique problem for the interpretation of self-colocation cases that I am recommending, since the same argument can be run on the assumption that Casper is genuinely selfcolocated. In fact, this problem has been the focus of attention in most previous discussions of self-colocation (Gilmore 2004, Carroll 2011, Wasserman 2018, Simon 2019). But one might worry that my account of what is going on in Casper's situation makes this problem much harder to solve.$^{30}$ For example, I can't say that velocity v1 is instantiated by a different Casper-shaped object than v2 is, since I've argued that there is just one Casper-shaped object at $\mathrm{r} 1$ at $\mathrm{t} 2$. Nor can I relativize v1 and v2 to different hyperlocations or personal times, since I rejected similar appeals above. So what is the endurantist to do?

My preferred solution to this problem was first suggested - but not endorsed - by Sider $(2001,102)$ and was later advocated by Wasserman (2005 \& 2018, 203) and Carroll (2011, 366-9). Since it has been discussed before, I will be brief about it here. The strategy is to deny the premise that, if younger Casper has velocity v1, then he does not have velocity v2. Even without relativizing these velocities to different indices of some sort - a strategy that both Carroll and Wasserman consider and reject - this premise is neither a logical truth nor a consequence of Leibniz's law. So there is room to contend, as Carroll and Wasserman do, that certain apparently incompatible pairs of properties like different bodily positions, weights, and velocities turn out to be compatible after all. Both authors argue that

\footnotetext{
${ }^{29}$ Cf. Wasserman's (2018, 200-203) discussion of a self-colocated boson.

${ }^{30} \mathrm{~A}$ referee raised this worry.
} 
the properties in each pair, though not co-instantiated in ordinary circumstances, are co-instantiated in certain unusual circumstances, but we tend to generalize from the former and so mistakenly conclude that the property pairs are incompatible. In reality, they are not incompatible at all.

So I think the endurantist should deny that Casper is self-colocated. More generally, the cases in the literature which have been dubbed cases of self-colocation or spooky coincidence are not, in fact, special cases of colocation or coincidence at all. They are merely cases of singular location in strange contexts.

Moreover, the endurantist can offer a plausible debunking story about why it seems to some philosophers that cases like Casper's are cases of self-colocation. For it's natural to think of spatially separated objects with apparently incompatible properties as distinct objects, even if, in the special case of a backward time traveler, the endurantist will say that they are not distinct after all. For that reason, it is easy slip into thinking about younger and older Casper as though they were distinct objects, and therefore younger Casper colocates with older Casper when older Casper passes through him. So, the idea that Casper self-colocates is ultimately based on a natural and tempting mistake in reasoning about backward time travelers.

I conclude that the endurantist can give an adequate account of what is going on in cases of apparent self-colocation. But to do so, she must deny that apparent cases of self-colocation are genuine cases of self-colocation. ${ }^{31}$

\footnotetext{
${ }^{31}$ Thanks to Joshua Spencer, who helped me to develop the colocation puzzle and served as my mentor throughout the project. His suggestions show up throughout the paper in too many places to tag individually. Thanks to Phil Bricker, Joshua Spencer, and a referee for helping me to see that the puzzle could be framed as a problem primarily for the endurantist. Thanks also to Cody Gilmore, Stan Husi, William Wainwright, Blain Neufeld, the participants in Blain's 2016 writing workshop, and various referees and editors for helpful discussion and/or written comments. Again, their suggestions show up throughout the paper in too many places to tag individually.
} 


\section{References}

Barnette, R. L. (1978). Does Quantum Mechanics Disprove the Principle of the Identity of Indiscernibles? Philosophy of Science 45(3): 466-470. DOI:10.1086/288822

Brower, J. E. (2010). Aristotelian Endurantism: A New Solution to the Problem of Temporary Intrinsics. Mind 119 (476): 883-905. DOI: $10.1093 / \mathrm{mind} / \mathrm{fzq} 072$.

Brzozowski, J. (2008). On Locating Composite Objects. In D. Zimmerman (Ed.), Oxford Studies in Metaphysics Volume 4. Oxford: Oxford University Press. 193-222.

Carroll, J. W. (2011). A Self-Visitation, Traveler Time, and Compatible Properties. Canadian Journal of Philosophy 41(3): 359-370. DOI: 10.1353 / cjp.2011.0025

Cortes, A. (1976). Leibniz's Principle of the Identity of Indiscernibles: A False Principle. Philosophy of Science 43(4): 491-505. DOI:10.1086/288707

Gilmore, C. (2004). Material Objects: Metaphysical Issues. Doctoral dissertation at Princeton University.

Gilmore, C. (2014). "Location and Mereology", The Stanford Encyclopedia of Philosophy (Fall 2014 Edition), Edward N. Zalta (ed.), URL = $<$ http:/ / plato.stanford.edu/archives/fall2014/entries/locationmereology/>.

Ginsberg, A. (1981) Quantum Theory and the Identity of Indiscernibles Revisited. Philosophy of Science 48(3): 487-491. DOI: 10.1086/ 289012

Haslanger, S. (2003). Persistence Through Time. In M. J. Loux and D. Zimmerman (Eds.), The Oxford Handbook of Metaphysics. Oxford: Oxford University Press, pp. 315-354.

Hawley, K. (2001). How Things Persist. Oxford: Oxford University Press.

Hudson, H. (2005). The Metaphysics of Hyperspace. Oxford: Oxford University Press.

Hudson, H. (2008a) Précis of The Metaphysics of Hyperspace. Philosophy and Phenomenological Research76(2): 422-426. 
Hudson, H. (2008b) Reply to Parsons, Reply to Heller, and Reply to Rea. Philosophy and Phenomenological Research 76(2): 452-470.

Hudson, H. (2014). The Fall and Hypertime. Oxford: Oxford University Press. Johnston, M. (1984). Particulars and Persistence. Doctoral dissertation at Princeton University.

Kleinschmidt, S. (2017). At It Again: Time Travel and the At-At Account of Motion. Erkenntnis 82(1): 185-198 (2017).

Kleinschmidt, S. (2014). Mereology and Location. Oxford: Oxford University Press.

Kurtz, R. (2006). Introduction to Persistence: What's the Problem? In S. Haslanger and R. Kurtz (Eds.), Persistence: Contemporary Readings. Cambridge, MA: MIT Press.

Lewis, D. (1986). On the Plurality of Worlds. Oxford: Basil Blackwell.

Lewis, D. (1976). The Paradoxes of Time Travel. American Philosophical Quarterly 13(2): 145-152.

McDaniel, K. (2007b). Extended Simples. Philosophical Studies 133(1): 131141. DOI: $10.1007 /$ s11098-006-9010-y

Parsons, J. (2008). Review: Hudson on Location. Philosophy and Phenomenological Research 76(2): 427-435. DOI: ppr200876232

Pruss, Alexander R. (2017). Self-Colocation. Alexander Pruss's Blog, April 6, 2017. <http://alexanderpruss.blogspot.com/2017/04/selfcolocation.html>.

Saucedo, R. (2011). Parthood and Location. In K. Bennett and D. Zimmerman (Eds.), Oxford Studies in Metaphysics Vol. 6 Oxford: Oxford University Press 2011, pp. 125-284.

Sider, T. (2000). Simply Possible. Philosophy and Phenomenological Research 60: 585-590. DOI: $10.2307 / 2653816$

Sider, T. (2001). Four-Dimensionalism: An Ontology of Persistence and Time. Oxford: Oxford University Press.

Simon, J. (2019). Fragmenting the Wave Function. Oxford Studies in Metaphysics Volume 11. In K. Bennett and D. Zimmerman (Eds.), Oxford: Oxford University Press: 123-147. 
Teller, P. (1983). Quantum Physics, the Identity of Indiscernibles, and Some Unanswered Questions. Philosophy of Science 50(2): 309-319. DOI:10.1086/289112

Wasserman, R. (2005). The problem of Change. Doctoral dissertation at Rutgers University.

Wasserman, R. (2018). Paradoxes of Time Travel. Oxford: Oxford University Press. 